\title{
Calibration of the global physical activity questionnaire to Accelerometry measured physical activity and sedentary behavior
}

Kristen M. Metcalf ${ }^{1 *}$ (D, Barbara I. Baquero ${ }^{2}$, Mayra L. Coronado Garcia ${ }^{3}$, Shelby L. Francis ${ }^{4}$, Kathleen F. Janz ${ }^{5}$, Helena H. Laroche ${ }^{6}$ and Daniel K. Sewell ${ }^{7}$

\begin{abstract}
Background: Self-report questionnaires are a valuable method of physical activity measurement in public health research; however, accuracy is often lacking. The purpose of this study is to improve the validity of the Global Physical Activity Questionnaire by calibrating it to 7 days of accelerometer measured physical activity and sedentary behavior.

Methods: Participants $(n=108)$ wore an ActiGraph GT9X Link on their non-dominant wrist for 7 days. Following the accelerometer wear period, participants completed a telephone Global Physical Activity Questionnaire with a research assistant. Data were split into training and testing samples, and multivariable linear regression models built using functions of the GPAQ self-report data to predict ActiGraph measured physical activity and sedentary behavior. Models were evaluated with the testing sample and an independent validation sample $(n=120)$ using Mean Squared Prediction Errors.

Results: The prediction models utilized sedentary behavior, and moderate- and vigorous-intensity physical activity self-reported scores from the questionnaire, and participant age. Transformations of each variable, as well as break point analysis were considered. Prediction errors were reduced by $77.7-80.6 \%$ for sedentary behavior and 61.3-98.6\% for physical activity by using the multivariable linear regression models over raw questionnaire scores.

Conclusions: This research demonstrates the utility of calibrating self-report questionnaire data to objective measures to improve estimates of physical activity and sedentary behavior. It provides an understanding of the divide between objective and subjective measures, and provides a means to utilize the two methods as a unified measure.
\end{abstract}

Keywords: Accelerometry, Calibration, Physical activity, Sedentary behavior, Self-report, Measurement, Public health

\section{Background}

Physical activity (PA) is important as an exposure and outcome variable in public health research. Although objective measurement of PA using accelerometry is widely used in public health research, its costs, complexities, and participant burden preclude its use in all studies. As such, self-report measures of PA, including questionnaires, remain a valuable method of PA measurement that

\footnotetext{
* Correspondence: Kristen-metcalf@uiowa.edu

'University of lowa, 102 Field House, lowa City, IA 52242, USA

Full list of author information is available at the end of the article
}

can provide unique and valuable information about domains in addition to estimates of volume of PA. Furthermore, self-report measures of PA allow for comparison of longitudinal data where objective measures were not utilized in all measurement periods. When compared to accelerometry, the most commonly used self-report measures of PA, e.g., the International Physical Activity Questionnaire, the Global Physical Activity Questionnaire (GPAQ), and the Seven-Day Physical Activity Recall show low to moderate validity of $0.17-0.41[1-4]$. Clearly, there is a need to improve the accuracy of PA self reports. For these reasons, 
many public health research studies utilize both objective and self-report measures of PA, such as the National Health and Nutrition Examination Survey, the Women's Health Study, and the Iowa Bone Development Study [5-7].

The GPAQ was developed by the World Health Organization (WHO) for PA surveillance, including the assessment of PA trends over time [8]. The GPAQ is available in nine languages, and has been utilized as a part of the WHO STEPwise Approach to Non-Communicable Chronic Disease Risk Factor Surveillance in over 100 countries [9]. The GPAQ collects information about PA in three domains: work, travel, and recreation, as well as average time per day spent in sedentary behavior (i.e., sitting and reclining) [10]. It is scored in minutes per day $(\mathrm{min} / \mathrm{d})$ to provide meaningful behavioral units, rather than a unit-less questionnaire score that is difficult to interpret, e.g., the Godin-Shephard Leisure-Time Exercise Questionnaire [11]. The first version of the GPAQ was validated in multiple populations, including adults from the Bangladesh, Brazil, China, Ethiopia, India, Indonesia, Japan, Portugal, South Africa, the United States, and Vietnam. The GPAQ had low to moderate validity for total PA when compared to pedometers $(r=0.31-0.39)$ [8, $12,13]$ and accelerometers $(r=0.20-0.34)$ [14]. Validity appeared lower for vigorous-intensity PA $(r=0.23-0.26)$. However, the first version of the GPAQ showed good testretest reliability $(r=0.67-0.81)[8,12]$. This suggests that while the GPAQ may not be appropriate for crosssectional studies due to its low to moderate validity, it may be a good tool for assessing changes in PA over time.

The GPAQ was revised to improve wording and shorten its length. Herrmann and colleagues examined the second version's validity by comparing it to PA measured using the ActiGraph accelerometer and the Yamax pedometer. In adults, the GPAQ had a low level of agreement with the ActiGraph for moderate- and vigorous-intensity PA (MVPA) $(r=0.26)$ and a moderate level of agreement with the Yamax pedometer for steps/ day $(r=0.39)$. Associations were negative for sedentary behavior, with a non-significant level of agreement between the GPAQ and ActiGraph $(r=-0.12)$. However, once again, the GPAQ showed good short-term reliability, with coefficients ranging from $r=0.83-0.96$ [2]. Cleland and colleagues assessed the validity of the second version of the GPAQ in adults living in the United Kingdom, and reported a moderate level of agreement between the GPAQ and ActiGraph measured PA $(r=0.48)$. The GPAQ had a moderate, but non-significant agreement with the ActiGraph for change over time in MVPA $(r=0.52)$ and a non-significant negative agreement of change over time in sedentary behavior $(r=-0.02)$. These researchers also found a negative bias with the GPAQ, indicating that individuals who were more active, were more likely to over-report PA [3].
Recently Saint-Maurice and colleagues demonstrated a calibration strategy to improve interpretability of the Physical Activity Questionnaire for Children and Physical Activity Questionnaire for Adolescents. They simultaneously measured PA in children and adolescents with questionnaires and accelerometers, then used multivariable linear regression to calibrate the relationship between questionnaire scores and ActiGraph measured MVPA [15]. Welk and colleagues developed a similar method to adjust for error in the 24-Hour Physical Activity Recall (PAR) in adults, by simultaneously measuring PA with the 24-Hour PAR and the SenseWear Armband Mini [16]. The 24-h PAR queries contextual PA information like that of the GPAQ (e.g., domain), however it takes on average $20 \mathrm{~min}$ to complete, and inquires about the previous $24 \mathrm{~h}$. These studies showed the feasibility of calibrating self-reported PA data to objectively measured PA, and the ability to improve their accuracy and usability. The current study used a similar strategy to provide a unique way to calibrate and compare past and present self-reported PA measures with objective measures, which are the current standard for PA measurement. It also allowed for better understanding of the direction and level of disconnect between selfreported and objectively measured PA. The purpose of this study was to improve the validity of the GPAQ for measuring PA and sedentary behavior by leveraging all information from the self-reported data in a prediction model trained on accelerometer measures in a random sample of mainly white and Latino adults, and to further test this calibration in an independent cohort of white, African American, and Latino adults.

\section{Methods}

\section{Participants}

Data for this study were collected between November 2015 and May 2016 as a part of baseline data collection for Active Ottumwa (AO). AO is a community-based participatory research project designed to increase the PA of all residents of Ottumwa, Iowa through the activation of Lay Health Advisors as Physical Activity Leaders. Ottumwa is a city in southeast Iowa with a population around 25,000 , of which $12 \%$ are Hispanic or Latino. Ottumwa is largely a processing and manufacturing community [17].

Participants of AO are a random sample of Ottumwa residents $(n=139)$, who were recruited via random digit dialing. The study recruited additional Latino residents via Respondent Driven Sampling [18]. Eligibility requirements included (1) being at least 18 years of age or older; (2) having lived in Ottumwa for at least 6 months; (3) planning to stay in Ottumwa for at least 2 years; and (4) passing physical functioning screening questions modified from NHANES [19]. 


\section{Procedures}

Individuals who were interested in participation attended a baseline data collection visit at the $\mathrm{AO}$ office with a bilingual (English-Spanish) data collector. Participants completed a health survey, and anthropometric measurements. Upon completion of the office visit, participants were fitted with an ActiGraph GT9X Link (ActiGraph LLC, Pensacola, FL) accelerometer, which served as the criterion measure of PA. The ActiGraph GT9X Link is a small $(3.5 \times 3.5 \times 1 \mathrm{~cm})$, lightweight (14 g), and waterproof tri-axial accelerometer. Its primary accelerometer has a dynamic range of \pm 8 gravitational units, and was initialized to collect data at a sampling rate of $80 \mathrm{~Hz}$. The accelerometer was attached to the participants' non-dominant wrist using a hospital band, and was worn continuously for 7 days, including at night. Participants were instructed to remove the accelerometer 8 days following their office visit, and return it via pre-paid mail.

On the accelerometer removal day, a research assistant contacted the participants and administered the GPAQ over the phone. The GPAQ asked participants to recall their PA and sedentary behavior over the past 7 days, to coincide with the time the accelerometer was worn. The GPAQ was designed to fit the specific population being assessed through the use of tailored examples. Within each PA domain, participants were asked to report time spent in moderate-intensity and vigorous-intensity PA. Participants were also queried about sedentary behavior by reporting time spent sitting or reclining. The GPAQ was scored by calculating the average time per day spent in each activity domain and intensity.

\section{Independent sample validation}

The GPAQ calibration models were tested on an independent sample representing a dissimilar population. The validation sample data were collected between December 2014 and September 2016 as a part of baseline data for the Living Well Together (LWT) study in Polk County, Iowa. Participants of the LWT study were low income, urban, obese adults $\left(\mathrm{BMI} \geq 30 \mathrm{~kg} / \mathrm{m}^{2}\right)$ who were the primary caregiver of a child six to 12 years old, and were drawn from an overall study population that was 29\% African American, 25\% Latino, and 41\% white.

Similar data collection procedures were followed for the LWT study, as for AO. Participants completed baseline data collection and were fitted with an ActiGraph GT9X Link accelerometer on their non-dominant wrist using a hospital band. Participants wore the accelerometer for seven consecutive days, including nights, and were asked to remove it on the eighth day following their baseline data collection visit. A research team member contacted the participants via telephone on the eighth day to complete the GPAQ, where they were queried about their PA over the preceding 7 days.

\section{Data processing}

For both samples, upon receipt of the accelerometer by the research team, data were downloaded and screened for wear-time and monitor malfunction. Participant sleep time was manually extracted using the Physical Activity and Health Outcomes Laboratory sleep algorithm (University of Iowa, Iowa City, IA; details available from corresponding author). Sedentary behavior $(\mathrm{min} / \mathrm{d})$ was extracted using the Staudenmayer decision tree algorithm [20] programmed into $\mathrm{R}$ software (version 3.3.1). Moderate-, and vigorous-intensity PA $(\mathrm{min} / \mathrm{d})$ were calculated using the Hildebrand algorithm [21] in a Visual Basic analysis program developed at the University of Iowa (R. Paulos, University of Iowa, Iowa City, IA). Accelerometry data were matched with the corresponding GPAQ data for analysis. Participants were excluded if they had less than four valid days of accelerometer data collection. A valid day was defined as having at least $10 \mathrm{~h}$ of awake data collection.

\section{Statistical analyses}

The analytical goal was to take the information included in the self-reported GPAQ and construct prediction models using each of the outcomes from the accelerometry data as the response variable. To this end, four multiple linear regression models were trained corresponding to sedentary behavior, moderate-intensity PA, vigorous-intensity PA, and MVPA ( $\mathrm{min} / \mathrm{d}$ ) as measured by ActiGraph. Covariates for each of the four models included gender, age, and self-reported $\mathrm{min} / \mathrm{d}$ of sedentary behavior, moderate-intensity PA, vigorous-intensity PA, and the sum of moderate- and vigorous-intensity PA corresponding to the GPAQ scores. Due to the lack of a clear linear relationship between these variables and the ActiGraph data (correlations between ActiGraph and corresponding GPAQ values ranged from 0.04 to 0.19 ), several transformations of each variable were considered, namely log, square root, linear, and quadratic relationships. Importantly, our purpose here is not to estimate and make inference on the relationship between the GPAQ self-report outcomes and the corresponding outcomes as measured by accelerometry, but rather to take the self-report outcomes and some easy to measure demographic variables and make accurate predictions of the outcomes as would be measured by accelerometry.

In addition to the four covariate transformations mentioned above, break point analysis was also considered. Break point analysis entails estimating from the data a change point in the covariate space where the linear relationship between the response and the covariate changes [22]. That is, at a break point the intercept and the slope for the covariate under consideration changes. This method works by adding a covariate binary 
indicator which equals one if the variable under consideration is greater than its break point and zero otherwise; the interaction of this binary indicator with the variable itself is also considered. For each continuous covariate and for each of the four response variables, a bivariate break point analysis was performed and the ensuing non-linear relationship was considered to be the fifth possible covariate transformation.

AO data were split into training (80\%) and testing (20\%) samples. The training sample was used to create the multiple linear regression models. Akaike Information Criterion (AIC) was used to perform model selection on the models created based on the training sample. Specifically, for each of the four responses, an exhaustive search over the $2 \times 6^{5}(=15,552)$ candidate models was performed, and for each model fit the AIC was computed, thus giving a measure of predictive ability for each of the 15,552 models for each of the four outcomes [23]. The model with the best AIC on the training data was selected for each outcome and subsequently evaluated on the out of sample data. Mean Squared Prediction Errors (MSPE) were calculated for the $\mathrm{AO}$ testing and LWT independent validation samples. Percent reductions in MSPE were evaluated to measure error relative to the raw GPAQ estimates of sedentary behavior and PA.

In addition to the analysis described above, for comparative purposes we implemented a simple outcome-matching model only utilizing gender, age, and the matching GPAQ outcome, e.g., using gender, age, and self-reported sedentary behavior to predict ActiGraph measured sedentary behavior. All analyses were performed using $\mathrm{R}$ software (version 3.3.1). Break point analyses were performed using the strucchange package [24].

\section{Results}

\section{Participants}

The AO sample used in our analysis included 108 participants ( $n=74$ females; $n=34$ males). Average age of participants was 49.4 years (range: 19.8-68.7 years). Participants wore the ActiGraph accelerometer for an average of 7 days (range: 4-10 days) and had an average awake wear time of $980 \mathrm{~min} / \mathrm{d}(16.3 \mathrm{~h})$. Participants accumulated $72.8 \mathrm{~min} / \mathrm{d}$ of MVPA on average, measured with the ActiGraph, and reported an average of 104.4 $\mathrm{min} / \mathrm{d}$ of MVPA on the GPAQ. Model-adjusted GPAQ MVPA was $74.9 \mathrm{~min} / \mathrm{d}$. Participants had $543.2 \mathrm{~min} / \mathrm{d}$ of sedentary behavior measured with the ActiGraph, and reported an average of $338.9 \mathrm{~min} / \mathrm{d}$ of sedentary behavior on the GPAQ. Model-adjusted GPAQ sedentary behavior was $534.8 \mathrm{~min} / \mathrm{d}$. Raw GPAQ scores $(\mathrm{min} / \mathrm{d})$ were weakly correlated with ActiGraph measured PA ( $r=0.04$ for moderate-intensity PA; $r=0.19$ for vigorous-intensity PA) and sedentary behavior $(r=0.19)$.
Table 1 Descriptive Statistics of Active Ottumwa Training and Testing Samples and Living Well Together Validation Sample

\begin{tabular}{|c|c|c|c|}
\hline & $\begin{array}{l}\text { Active Ottumwa } \\
\text { Training Sample } \\
(n=86) \\
\text { Mean (SD) }\end{array}$ & $\begin{array}{l}\text { Active } \\
\text { Ottumwa } \\
\text { Testing } \\
\text { Sample } \\
(n=22) \\
\text { Mean (SD) }\end{array}$ & $\begin{array}{l}\text { Living Well } \\
\text { Together } \\
\text { Validation } \\
\text { Sample } \\
(n=120) \\
\text { Mean (SD) }\end{array}$ \\
\hline Age (year) & $49.1(14.1)$ & $50.6(13.7)$ & $36.5(8.1)$ \\
\hline Gender (\% female) & 67.4 & 72.7 & 92.5 \\
\hline \multicolumn{4}{|l|}{ Race (\%) } \\
\hline White & 81.4 & 86.4 & 42.2 \\
\hline Latino & 14.0 & 9.1 & 23.5 \\
\hline African American & 3.5 & 4.5 & 23.5 \\
\hline Other & 1.1 & 0.0 & 10.8 \\
\hline Height (cm) & $164.9(9.20)$ & $165.2(9.75)$ & $163.4(10.8)$ \\
\hline Weight (kg) & $85.5(23.8)$ & $87.7(25.8)$ & $108.3(25.6)$ \\
\hline BMI $\left(\mathrm{kg} / \mathrm{m}^{2}\right)$ & $31.2(7.8)$ & $32.1(8.64)$ & $40.8(11.3)$ \\
\hline $\begin{array}{l}\text { ActiGraph moderate- } \\
\text { intensity PA (min/d) }\end{array}$ & $74.0(61.8)$ & $60.8(46.0)$ & $82.0(56.6)$ \\
\hline $\begin{array}{l}\text { GPAQ moderate- } \\
\text { intensity PA (min/d) }\end{array}$ & $72.8(95.5)$ & $91.9(193.9)$ & $47.2(77.0)$ \\
\hline $\begin{array}{l}\text { ActiGraph vigorous- } \\
\text { intensity PA (min/d) }\end{array}$ & $0.97(2.13)$ & $3.29(11.7)$ & 1.69 (5.69) \\
\hline $\begin{array}{l}\text { GPAQ vigorous- } \\
\text { intensity PA (min/d) }\end{array}$ & $23.9(47.9)$ & $42.8(85.9)$ & $21.6(44.7)$ \\
\hline $\begin{array}{l}\text { ActiGraph sedentary } \\
\text { behavior }(\mathrm{min} / \mathrm{d})\end{array}$ & $535.1(117.8)$ & $574.6(154.4)$ & $510.7(211.1)$ \\
\hline $\begin{array}{l}\text { GPAQ sedentary } \\
\text { behavior }(\mathrm{min} / \mathrm{d})\end{array}$ & $332.7(208.0)$ & $363.2(210.4)$ & $294.9(201.0)$ \\
\hline
\end{tabular}

$S D=$ standard deviation; $\mathrm{cm}=$ centimeters; $\mathrm{kg}=$ kilograms; $m=$ meters; $P A=$ physical activity; $\min / d=$ minutes per day; $G P A Q=$ Global Physical Activity Questionnaire

The LWT independent validation sample included 120 participants ( $n=111$ females; $n=9$ males). Average age of participants was 36.5 years (range: 23.4-62.3 years). Participants wore the ActiGraph accelerometer for an average of 7 days (range: 4-10 days) and had an average awake wear time of $984 \mathrm{~min} / \mathrm{d}(16.4 \mathrm{~h})$. Participants

Table 2 Percent of Reported Moderate- and Vigorous-Intensity Physical Activity Spent in Each Activity Domain

\begin{tabular}{llll}
\hline & $\begin{array}{l}\text { Active Ottumwa } \\
\text { Training } \\
\text { Sample }(n=86)\end{array}$ & $\begin{array}{l}\text { Active } \\
\text { Ottumwa } \\
\text { Testing } \\
\text { Sample } \\
(n=22)\end{array}$ & $\begin{array}{l}\text { Living Well } \\
\text { Together } \\
\text { Validation } \\
\text { Sample } \\
(n=120)\end{array}$ \\
\hline Work (\%MVPA) & 63.89 & 78.17 & 36.76 \\
Travel (\%MVPA) & 5.45 & 1.65 & 13.21 \\
Recreation (\%MVPA) & 30.66 & 20.18 & 50.03 \\
\hline
\end{tabular}

$\% \mathrm{MVPA}$ - percentage of total reported daily moderate- and vigorous-intensity physical activity 
a

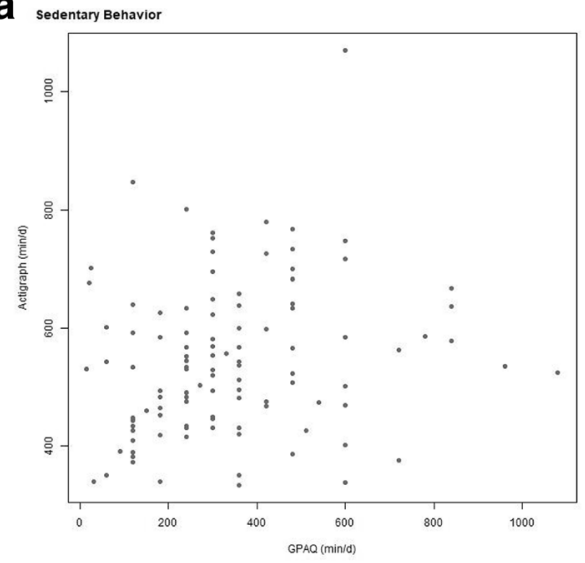

C

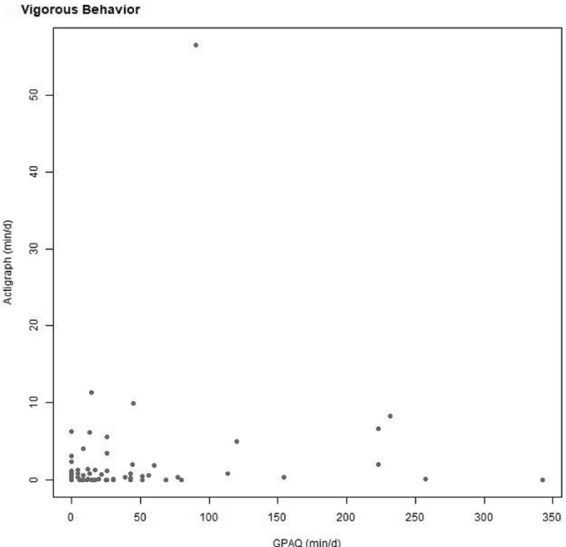

b

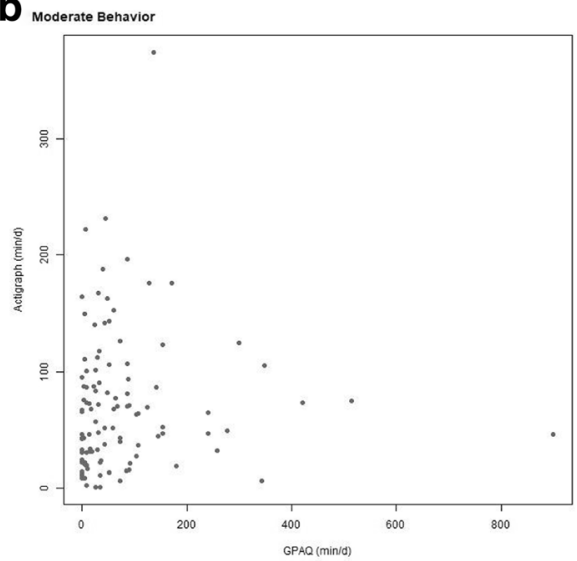

$\mathbf{d}_{\text {mo }}$

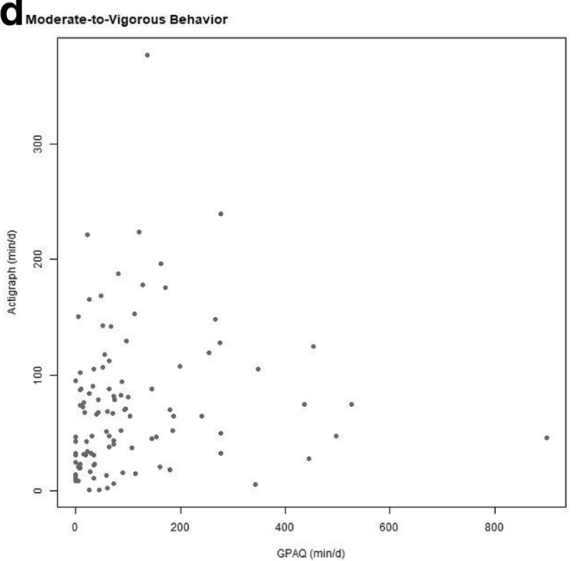

Fig. 1 Global Physical Activity Questionnaire Reported and ActiGraph Measured Sedentary Behavior and Physical Activity. Raw GPAQ reported sedentary behavior and PA (min/d) plotted against ActiGraph measured sedentary behavior and PA (min/d)

accumulated $83.7 \mathrm{~min} / \mathrm{d}$ of MVPA on average, measured with the ActiGraph, and reported an average of $68.8 \mathrm{~min} / \mathrm{d}$ of MVPA on the GPAQ. Model-adjusted GPAQ MVPA was $84.8 \mathrm{~min} / \mathrm{d}$. Participants had $510.7 \mathrm{~min} / \mathrm{d}$ of sedentary behavior measured with the ActiGraph, and reported an average of $294.9 \mathrm{~min} / \mathrm{d}$ of sedentary behavior on the GPAQ. Model-adjusted GPAQ sedentary behavior was $545.3 \mathrm{~min} / \mathrm{d}$. Table 1 shows the descriptive statistics of the testing, training, and independent validation samples. Table 2 shows the

Table 3 Proportion of Variance Explained for the OutcomeMatching Models and the Proposed Calibration Models

\begin{tabular}{|c|c|c|}
\hline Outcome & $\begin{array}{l}R^{2} \text { for Outcome-Matching } \\
\text { Model }\end{array}$ & $\begin{array}{l}R^{2} \text { for Final } \\
\text { Calibration } \\
\text { Model }\end{array}$ \\
\hline Sedentary behavior & 0.025 & 0.097 \\
\hline Moderate-intensity PA & 0.144 & 0.341 \\
\hline Vigorous-intensity PA & 0.177 & 0.364 \\
\hline MVPA & 0.156 & 0.282 \\
\hline
\end{tabular}

$P A=$ physical activity $M V P A=$ moderate- and vigorous-intensity physical activity breakdown of GPAQ reported MVPA by domain. The table demonstrates the vast differences in how each sample accrued daily MVPA. The AO sample accumulated the majority of MVPA from work-related activity (66.4\%), while the LWT sample accumulated the majority of MVPA from recreation (50.0\%). Additionally, the LWT sample had more MVPA from travel than the AO samples.

\section{Calibration}

We developed multiple linear regression models to the AO training set utilizing as covariates gender, age, GPAQ reported sedentary behavior, GPAQ reported moderateintensity PA, GPAQ reported vigorous-intensity PA, and GPAQ reported MVPA to predict accelerometer measured sedentary behavior and PA. Figure 1 shows the scatterplots of the self-reported GPAQ outcomes and the corresponding accelerometry outcome. This figure shows that there is a weak association between the self-report and accelerometry data. Table 3 corroborates this by giving the proportion of variance explained $\left(R^{2}\right)$ for the outcome-matching model using only gender, age, and the matching GPAQ outcome 
Table 4 Multiple Linear Regression Prediction Models for ActiGraph Measured Sedentary Behavior and Physical Activity

\begin{tabular}{|c|c|c|}
\hline Model Parameters & Estimate & $\begin{array}{l}\text { Break Factor } \\
\text { Cutoff }\end{array}$ \\
\hline \multicolumn{3}{|l|}{ Sedentary Behavior } \\
\hline Intercept & 616.5 & \\
\hline GPAQ Moderate-Intensity PA & -0.03 & \\
\hline GPAQ Moderate-Intensity PA Break Factor & -78.0 & 0.00 \\
\hline GPAQ Vigorous-Intensity PA & -0.45 & \\
\hline \multicolumn{3}{|l|}{ Moderate-Intensity PA } \\
\hline Intercept & 348.2 & \\
\hline SQRT GPAQ Sedentary Behavior & -1.87 & \\
\hline GPAQ Moderate-Intensity PA² & $1.65 \times 10^{-3}$ & \\
\hline GPAQ Vigorous-Intensity PA ${ }^{2}$ & $3.54 \times 10^{-3}$ & \\
\hline LOG Age & -65.3 & \\
\hline GPAQ MVPA & -0.07 & \\
\hline GPAQ MVPA Break Factor & 214.9 & 113.6 \\
\hline $\begin{array}{l}\text { Interaction: (GPAQ MVPA)*(MVPA Break } \\
\text { Factor) }\end{array}$ & -1.15 & \\
\hline \multicolumn{3}{|l|}{ Vigorous-Intensity PA } \\
\hline Intercept & 10.5 & \\
\hline GPAQ Moderate-Intensity PA² & $2.91 \times 10^{-5}$ & \\
\hline GPAQ Vigorous-intensity PA² & $7.84 \times 10^{-5}$ & \\
\hline Age & -0.29 & \\
\hline Age Break Factor & -9.77 & 27.6 \\
\hline GPAQ MVPA & -0.01 & \\
\hline GPAQ MVPA Break Factor & 2.14 & 38.6 \\
\hline Interaction: (Age)*(Age Break Factor) & 0.28 & \\
\hline $\begin{array}{l}\text { Interaction: (GPAQ MVPA)*(MVPA Break } \\
\text { Factor) }\end{array}$ & $-6.55 \times 10^{-3}$ & \\
\hline \multicolumn{3}{|l|}{ MVPA } \\
\hline Intercept & 540.8 & \\
\hline LOG GPAQ Sedentary Behavior & -12.8 & \\
\hline Age & -15.0 & \\
\hline Age Break Factor & -356.0 & 29.1 \\
\hline LOG GPAQ MVPA & 5.78 & \\
\hline Interaction: (Age)*(Age Break Factor) & 13.7 & \\
\hline
\end{tabular}

$G P A Q=$ Global Physical Activity Questionnaire; $P A=$ physical activity; SQRT $=$ square root; $M V P A=$ moderate- and vigorous-intensity physical activity; * $=$ interaction

variable as covariates, as well as that for the final calibration model chosen by AIC. The proposed calibration model shows considerable improvement in fit over the simpler outcome-matching model. Table 4 shows the model parameters for final calibration models selected by AIC. Note that the break factor for a particular variable corresponds to a change in the intercept of the regression model when that variable takes values larger than the break factor cutoff. Similarly, the interaction of a variable with its break factor
Table 5 Percent reduction in Mean Squared Prediction Errors for Active Ottumwa and Living Well Together Validation Samples From Proposed Calibration Models

\begin{tabular}{lll}
\hline Predicted Variable & $\begin{array}{l}\text { Active } \\
\text { Ottumwa } \\
\text { \% Reduction } \\
\text { in MSPE }\end{array}$ & $\begin{array}{l}\text { Living Well } \\
\text { Together } \\
\text { in MSPE }\end{array}$ \\
\hline Sedentary behavior (min/d) & $77.7 \%$ & $80.6 \%$ \\
Moderate-intensity physical activity (min/d) & $66.4 \%$ & $61.3 \%$ \\
$\begin{array}{l}\text { Vigorous-intensity physical activity (min/d) } \\
\text { Moderate- and vigorous-intensity physical }\end{array}$ & $98.3 \%$ & $98.6 \%$ \\
activity (min/d) & $94.2 \%$ & $77.5 \%$ \\
\hline
\end{tabular}

MSPE = Mean Squared Prediction Errors; $\min / d=$ minutes per day

corresponds to a change in the slope term when that variable exceeds the cutoff value. For example, in the model predicting moderate-intensity PA, if the sum of a subject's self-reported moderate- and vigorous-intensity PA (GPAQ MVPA) is less than or equal to 113.6, then the intercept of the prediction model is 348.2 and the slope corresponding to GPAQ MVPA is - 0.07, otherwise the intercept is 563.1 $(=348.2+214.9)$ and the slope for GPAQ MVPA is -1.22 $(=-0.07-1.15)$.

\section{Validation}

Predictive ability of the multiple linear regression models was assessed using the percent reduction of Mean Squared Prediction Errors (MSPE) from the modeladjusted GPAQ values versus the raw GPAQ values. The percent reductions in MSPE are shown in Table 5, indicating that the calibrated GPAQ values led to dramatic reductions in error. From the table we see that the MSPE was reduced by $77.7 \%$ when using the modeladjusted GPAQ values versus the raw GPAQ values for sedentary behavior in the $\mathrm{AO}$ validation sample. In the same sample, error was reduced by $66.4 \%$ for moderateintensity PA, 98.3\% for vigorous-intensity PA, and 94.2\% for MVPA. Similarly, MSPE reductions ranged from $61.3 \%$ to $98.6 \%$ in the LWT independent validation sample.

Figure 2 shows the improvement in predictive value of the GPAQ using the multiple linear regression models detailed in Table 4. The vertical axis represents ActiGraph measured (A) sedentary behavior; (B) moderate-intensity PA; (C) vigorous-intensity PA; and (D) MVPA in $(\mathrm{min} / \mathrm{d})$, and the horizontal axis represents the predicted values. The triangles represent selfreported raw GPAQ values $(\mathrm{min} / \mathrm{d})$ of each participant in the validation sample, while the circles represent the model-adjusted GPAQ values ( $\mathrm{min} / \mathrm{d})$. Self-reported raw GPAQ values are connected to their corresponding model-adjusted GPAQ value with a horizontal line. This figure indicates that the adjusted GPAQ values pull the 

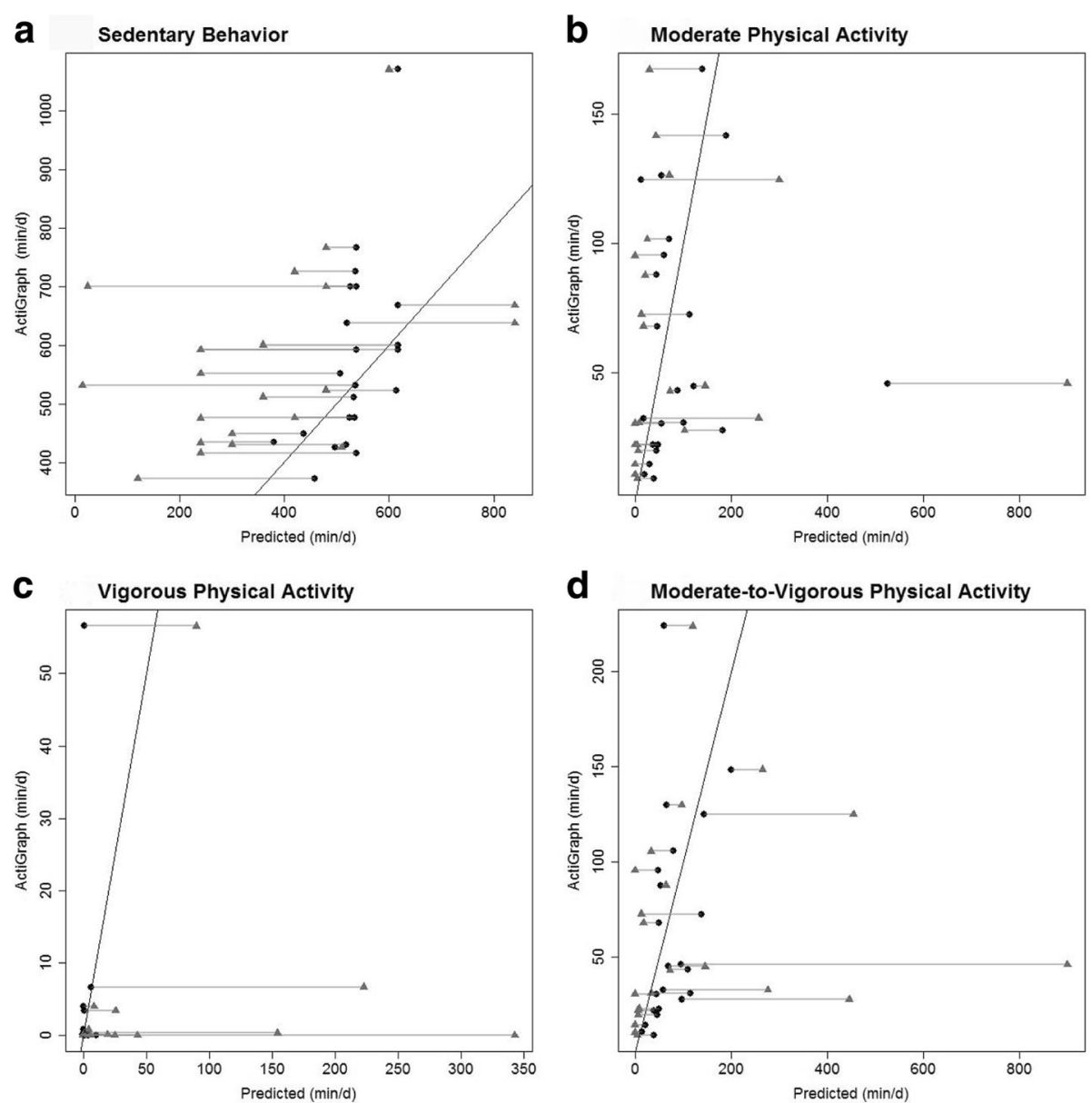

Fig. 2 Global Physical Activity Questionnaire Reported, Model-Adjusted, and ActiGraph Measured Sedentary Behavior and Physical Activity. Triangles = raw GPAQ reported sedentary behavior and PA (min/d) plotted against ActiGraph measured sedentary behavior and PA (min/d)Circles = model-adjusted sedentary behavior and PA ( $\mathrm{min} / \mathrm{d}$ ) plotted against ActiGraph measured sedentary behavior and PA (min/d). Self-reported raw GPAQ values are connected to their corresponding model-adjusted GPAQ value with a horizontal line. Covariates include sedentary behavior, moderate-intensity PA, vigorous-intensity PA, MVPA, and age.

raw GPAQ values closer to the truth (i.e., closer to the $y$ $=x$ line), thus showing the vast improvement in predictive accuracy of sedentary behavior and PA when using the multiple linear regression models over the raw GPAQ scores. Figure 3 shows these same plots but without the GPAQ values included. Despite the dramatic reduction in error, the predictions are still rather noisy. For the model predicting sedentary behavior, there seems to be an issue with obtaining relatively few unique predicted values; these two things imply that further work in this area would be beneficial.

\section{Discussion}

Calibrating PA questionnaires to objective measures of PA is a useful way to denoise this self-reported data. Additionally, this method can be utilized to compare self-reported PA data with objective measures, as well as better understand how these two measures can be used together. The current study demonstrates the feasibility of this approach by calibrating the GPAQ to 7 days of ActiGraph data.

The multiple linear regression models dramatically reduced prediction errors in both the AO testing sample and the LWT independent validation sample. Raw GPAQ scores overestimated MVPA by $31.6 \mathrm{~min} / \mathrm{d}$, and underestimated sedentary behavior by $204.3 \mathrm{~min} / \mathrm{d}$ in the $\mathrm{AO}$ sample using the ActiGraph as ground truth. While the model-adjusted mean GPAQ scores were within $2.1 \mathrm{~min} / \mathrm{d}$ and $8.4 \mathrm{~min} / \mathrm{d}$ of the ActiGraph measured mean MVPA and sedentary behavior, respectively. It is important to note that these reductions in error were seen in out-of-sample predictions, meaning they were applied to separate samples than what the models were built on. Additionally, using an independent 

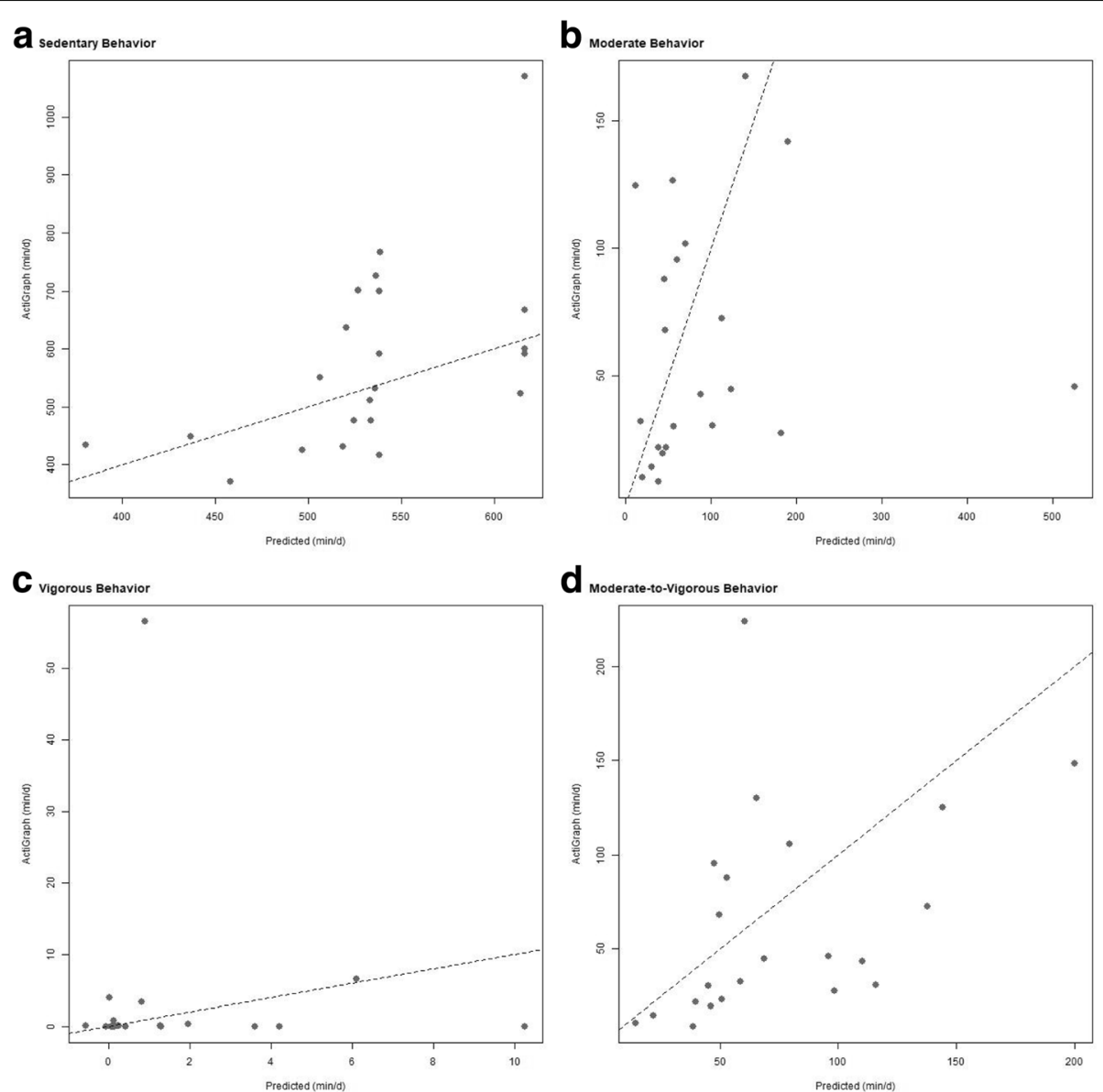

Fig. 3 Model-Adjusted and ActiGraph Measured Sedentary Behavior and Physical Activity. Model-adjusted sedentary behavior and PA (min/d) plotted against ActiGraph measured sedentary behavior and PA (min/d)

validation approach we were able to show the model's utility in a dissimilar population, who underreported PA rather than over-reported it, as the $\mathrm{AO}$ population did. The two populations also accumulated PA in differing domains; while the AO sample accumulated $66.4 \%$ of their MVPA from work-related activity, the LWT sample only accumulated $36.8 \%$ from work. The majority of the LWT sample's MVPA came from recreational activity (50.0\%), and they also accumulated substantially more travel-related MVPA than the AO sample (13.2\% for LWT versus $4.8 \%$ for AO). The LWT population has lower income and education levels than the AO population, and they are $42.4 \%$ White, $23.5 \%$ African American, and $23.5 \%$ Hispanic or Latino, while the AO population is $82.4 \%$ White, $3.7 \%$ African American, and $13.0 \%$ Hispanic or Latino. This provides some assurance of the calibration model's accuracy for new studies on dissimilar populations.

Adding age and performing data transformations on the self-reported GPAQ values improved PA prediction. Models utilized only data readily available from the questionnaire to improve usability in other studies where more thorough examinations, including anthropometric and objective PA data, are not available. The potential application of this calibration model includes improved predictions of PA in large sample surveillance data, longitudinal studies, and pre-/post-measurements where objective measures of PA are not feasible.

Strengths of the current study include the use of a population-wide random sample, as well as the use of data splitting to validate the models. Additionally, a major strength of the study lies in the ability to evaluate the predictive ability of our models on an independent sample from a disparate population with differing racial and socio-demographic make-up. The study utilizes one of the most commonly used PA questionnaires in current practice, which increases its usability for other researchers. Limitations of the study include a mostly white sample, while an effort was made to oversample the Latino population in Ottumwa. The study sample is also mostly female, particularly in the independent validation sample. Finally, the training data set was necessarily small, potentially limiting the range of PA observed to be smaller than that which might be observed in a larger study. 


\section{Conclusions}

The logistics related to large scale public health research make self-reported PA data an attractive option. This manuscript demonstrates a unique way to calibrate and compare self-reported questionnaire data to objective PA measures to improve accuracy of PA estimates, as well as provides a better understanding of the disconnect between the two. Importantly, error in MVPA estimates was reduced by up to $94.2 \%$. Additionally, it helps researchers to harmonize different measures of the same variable, and to understand how self-reported and objective measures can be used together to create a more cohesive PA measure. Ultimately, improved PA measurement will lead to better understanding of PA determinants, as well as the complex relationships between PA and health outcomes, and to assess intervention effectiveness. Researchers can utilize this calibration model for self-reported GPAQ data via a free Shiny web application [25].

\section{Abbreviations}

AIC: Akaike Information Criterion; AO: Active Ottumwa; cm: Centimeter; GPAQ: Global Physical Activity Questionnaire; kg: Kilogram; LWT: Living Well Together; m: Meter; min/d: Minutes per day; MSPE: Mean Squared Prediction Error; MVPA: Moderate- and vigorous-intensity physical activity; PA: Physical activity; PAR: Physical activity recall; SD: Standard deviation; SQRT: Square root; WHO: World Health Organization

\section{Acknowledgements}

The authors thank the study participants, and the Active Ottumwa staff for their work in completing this study. This publication was supported by Cooperative Agreement Number 1 U48 DP005021-01 from the Centers for Disease Control and Prevention. The findings and conclusions in this journal article are those of the authors and do not necessarily represent the official position of the Centers for Disease Control and Prevention.

\section{Funding}

This work was funded by the Centers for Disease Control and Prevention Cooperative Agreement Number 1 U48 DP005021-01, and the National Institutes of Health through grant 5R01 HL119882. The funding agencies were not involved in the study design, collection, analysis and interpretation of data, or in writing the manuscript

\section{Availability of data and materials}

The datasets used and analyzed during the current study are available from the corresponding author on reasonable request.

\section{Authors' contributions}

KM was a contributor in acquiring and interpreting data, and writing and revising the manuscript. MCG was a contributor in the analysis and interpretation of data, and writing and revising the manuscript. SF was a contributor in acquiring data, and writing and revising the manuscript. HL was a contributor in conception and design, and critically revising the manuscript for intellectual content. KJ was a contributor in conception and design, interpretation of data, and writing and revising the manuscript. BB was a contributor in conception and design, and critically revising the manuscript for intellectual content. DS was a contributor in conception and design, analysis and interpretation of data, and writing and revising the manuscript. All authors provided final approval of the manuscript and agree to be accountable for all aspects of the work.

\section{Ethics approval and consent to participate}

The Institutional Review Board at the University of lowa approved the study protocols and informed consent form. The IRB reference number is
201309708. All participants provided written informed consent prior to participating in study activities.

\section{Consent for publication}

Not applicable.

\section{Competing interests}

The authors declare that they have no competing interests.

\section{Publisher's Note}

Springer Nature remains neutral with regard to jurisdictional claims in published maps and institutional affiliations.

\section{Author details}

${ }^{1}$ University of lowa, 102 Field House, lowa City, IA 52242, USA. ${ }^{2}$ University of lowa, N418 CPHB, lowa City, IA 52242, USA. ${ }^{3}$ University of lowa, N300 CPHB, lowa City, IA 52242, USA. ${ }^{4}$ University of lowa, SW34 GH, lowa City, IA 52242, USA. ${ }^{5}$ University of lowa, E130 Field House, lowa City, IA 52242, USA.

'University of lowa, 280B MRF, lowa City, IA 52242, USA. ${ }^{7}$ University of lowa, N338 CPHB, lowa City, IA 52242, USA.

Received: 12 August 2017 Accepted: 12 March 2018

Published online: 27 March 2018

\section{References}

1. van der Ploeg HP, Tudor-Locke C, Marshall AL, Craig C, Hagstromer M, Sjostrom M, Bauman A. Reliability and validity of the International Physical Activity Questionnaire for Assessing Walking. Res Q Exerc Sport. 2010;81(1):97-101

2. Herrmann SD, Heumann KJ, Der Ananian CA, Ainsworth BE. Validity and reliability of the global physical activity questionnaire (GPAQ). Meas Phys Educ Exerc Sci. 2013;17:221-35

3. Cleland CL, Hunter RF, Kee F, Cupples ME, Sallis JF, Tully MA. Validity of the global physical activity questionnaire (GPAQ) in assessing levels and change in moderate-vigorous physical activity and sedentary behavior. BMC Public Health. 2014;14:1255-65.

4. Hayden-Wade HA, Coleman KJ, Sallis JF, Armstrong C. Validation of the telephone and in-person interview versions of the 7-day PAR. Med Sci Sports Exerc. 2003;35(5):801-9.

5. Zipf G, Chiappa M, Porter KS, Ostchega Y, Lewis BG, Dostal J. National Health and nutrition examination survey: plan and operations, 1999-2010. National Center for Health Statistics. Vital Health Stat. 2013;1(56):1-8.

6. Shiroma EJ, Cook NR, Manson JE, Buring JE, Rimm EB, Lee IM. Comparison of self-reported and accelerometer-assessed physical activity in older women. PLoS One. 2015;10(12):e0145950.

7. Janz KF, Medema-Johnson HC, Letuchy EM, Burns TL, Eichenberger Gilmore JM, Torner JC, Willing M, Levy SM. Subjective and objective measures of physical activity in relationship to bone mineral content during late childhood: the lowa bone development study. Br J Sports Med. 2008;42:658-63.

8. Armstrong T, Bull F. Development of the World Health Organization global physical activity questionnaire (GPAQ). J Public Health. 2006;14:66-70.

9. World Health Organization. Global physical activity surveillance. In: Chronic diseases and health promotion; 2016. http://www.who.int/chp/steps/GPAQ/ en/. Accessed 4 Dec 2016

10. Surveillance and Population-Based Prevention, Prevention of Noncommunicable Diseases Department. Global physical activity questionnaire (GPAQ) analysis guide. In: Chronic diseases and health promotion; 2016. http://www.who.int/chp/steps/ resources/GPAQ_Analysis_ Guide.pdf?ua=1. Accessed 4 Dec 2016.

11. Godin G, Shephard RJ. Godin Leisure-Time Exercise Questionnaire. Med Sci Sports Exerc. 1997;29(suppl 6):S36-8.

12. Bull FC, Maslin TS, Armstrong T. Global physical activity questionnaire (GPAQ): nine country reliability and validity study. J Phys Act Health. 2009;6: 790-804.

13. Thuy AB, Blizzard L, Schmidt M, Pham LH, Magnussen C, Dwyer T. Reliability and validity of the global physical activity questionnaire in Vietnam. J Phys Act Health. 2010;7:410-8

14. Trinh OTH, Nguyen ND, van der Ploeg HP, Dibley MJ, Bauman A. Test-retest repeatability and relative validity of the global physical activity questionnaire in a developing country context. J Phys Act Health. 2009; 6(Suppl 1):S46-53. 
15. Saint-Maurice PF, Welk GJ, Beyler NK, Bartee RT, Heelan KA. Calibration of self-report tools for physical activity research: the physical activity questionnaire (PAQ). BMC Public Health. 2014;14:461-9.

16. Welk GJ, Beyler NK, Kim Y, Matthews CE. Calibration of self-report measures of physical activity and sedentary behavior. Med Sci Sports Exerc. 2017;49(7): $1473-81$.

17. United States Census Bureau. https://www.census.gov/quickfacts/table/ BZA010215/ 1960465 (2016). Accessed 30 Apr 2017.

18. Heckathorn DD. Respondent driven sampling: a new approach to the study of hidden populations. Soc Probl. 1997;44(2):174-99.

19. Centers for Disease Control and Prevention. Physical functioning - PFQ. In: National Health and nutrition examination survey, 2015-2016 survey questionnaires. p. 2012. https://wwwn.cdc.gov/nchs/data/nhanes/20152016/questionnaires/PFQ_I.pdf. Accessed 2 May 2017.

20. Staudenmayer J, He S, Hickey A, Sasaki J, Freedson P. Methods to estimate aspects of physical activity and sedentary behavior from high-frequency wrist accelerometer measurements. J Appl Physiol. 2015;119:396-403.

21. Hildebrand M, Van Hees VT, Hansen BH, Ekelund U. Age group comparability of raw accelerometer output from wrist- and hip-worn monitors. Med Sci Sports Exerc. 2014;46(9):1816-24.

22. Zeileis A, Kleiber C, Draemer W, Hornik K. Testing and dating of structural changes in practice. Computational Statistics \& Data Analysis. 2003;44:109-23.

23. Shmueli G. To explain or to predict? Stat Sci. 2010;25(3):289-310.

24. Zeileis A, Leisch F, Hornik K, Kleiber C. Strucchange: an R package for testing for structural change in linear regression models. J Stat Softw. 2002;7(2):1-38.

25. Coronado Garcia, Mayra, Sewell DK. GPAQ to ActiGraph shiny web application. http://myweb.uiowa.edu/dksewell/GPAQToActiGraph.html. Accessed 4/25/2017.

\section{Submit your next manuscript to BioMed Central and we will help you at every step:}

- We accept pre-submission inquiries

- Our selector tool helps you to find the most relevant journal

- We provide round the clock customer support

- Convenient online submission

- Thorough peer review

- Inclusion in PubMed and all major indexing services

- Maximum visibility for your research

Submit your manuscript at www.biomedcentral.com/submit 